\title{
Un grand chimiste des lipides nous a quittés...
}

Michel Ollivon est décédé le 17 juin 2007.

Très connu et apprécié parmi les chercheurs en biochimie-nutrition et en lipochimie, souvent consulté et sollicité pour des conférences, des articles, pour sa maitrise et son inépuisable disponibilité, sa disparition a laissé consternés et émus tous ceux qui l'ont approché. Ses collaborateurs et amis de I'UMR du CNRS 8612 et Michel Parmentier au nom de l'AFECG et d'Eurofedlipid, ici en témoignent.

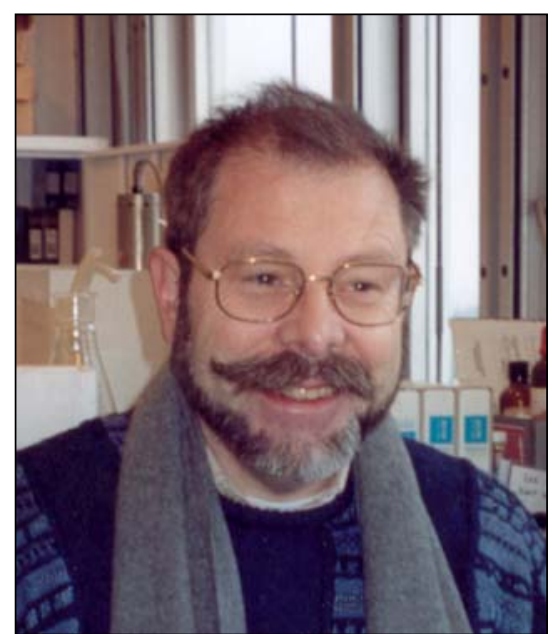

Michel Ollivon nous a quittés brutalement, au milieu de tous les siens.

Michel était d'abord un ami pour tous ceux, nombreux, qui l'ont côtoyé pendant sa carrière exemplaire. Arrivé parmi nous il y a près de 20 ans, il dirigeait l'équipe de physico-chimie des «systèmes polyphasés » et était incontestablement I'une des figures emblématiques de notre unité. Passionné et passionnant, il nous a acquis à la cause de la physico-chimie des lipides et à celle de la diffraction des rayons $X$ et a formé un grand nombre de jeunes « thésards».

Profondément humain, ce grand bonhomme de l'organisation supramoléculaire des lipides était un passionné de la recherche au point d'en perdre la notion du temps et de quitter souvent notre faculté, le soir, à des heures impossibles.

Michel se passionnait pour tout ce qui touche la manière dont les lipides s'auto-organisent et se structurent pour nous faciliter la vie, que ce soit pour découvrir de nouveaux systèmes de vectorisation des médicaments ou pour comprendre pourquoi le chocolat est si goûteux et le lait si utile. Combien de systèmes a-t-il décortiqué ?
Combien d'interactions a-t-il pisté ? Combien de structures bizarres et moins bizarres a-t-il trouvées?

II n'est point nécessaire de rechercher les citations ou autres impacts facteurs pour savoir que Michel était, en effet, un spécialiste reconnu de la physico-chimie des lipides tant au niveau national qu'international.

Pour ne citer que celui-ci, I'an dernier à Madrid, lors du $4^{\mathrm{e}}$ colloque $\mathrm{d}^{\prime}$ Eurofedlipid, la $\mathrm{SCl}$ anglaise lui avait conféré la prestigieuse « Hilditch lecture » en clôture du congrès européen. Pendant plus d'une heure, il enthousiasma son public, exposant magistralement comment les molécules lipidiques étaient capables de générer une infinie variété de structures aussi différentes que "des valises, des tuyaux, des aiguilles, des enveloppes... ».

Ses présentations étaient un moment de bonheur intellectuel pour son auditoire.

Ce grand chercheur était aussi un expérimentateur hors pair, capable de rendre reproductibles les phénomènes les plus fragiles. C'était un constructeur d'instruments de mesure plus originaux les uns que les autres qu'il mettait à la disposition de ses collaborateurs avec une grande ouverture.
À ce titre son dernier projet, la conception de I'appareil Microcalix permettant de coupler la calorimétrie différentielle à la diffraction des rayons $\mathrm{X}$ était un de ses sujets de fierté.

Nous l'aimions tous pour sa générosité car chacun sait qu'il adorait apporter sa contribution pour aider qui que ce soit, collègues statutaires de l'unité, thésards ou post-docs. II était toujours disponible pour essayer de résoudre, souvent avec succès, mais toujours avec une très grande gentillesse, n'importe quel problème scientifique ou technique, fut-t-il nécessaire d'utiliser le tournevis ou la clé à molette. Et combien $d$ 'industriels également ne I'auront-ils pas sollicité pour les aider à mieux comprendre tel ou tel comportement des phases lipidiques mises en œuvre!

Michel Ollivon à tous aura beaucoup donné. Sa disparition brutale laisse un grand vide dans la communauté des chercheurs.

P. Couvreur, Directeur de l'unité et toute l'équipe de Michel Ollivon : A. Angelova, C. Bourgaux, V. Faivre, G. Keller, G. Lebas et S. Lesieur, ainsi que Michel Parmentier (ancien président de l'AFECG, ancien président de l'EuroFedLipid) 